\title{
Study of the solar wind coupling to the time difference horizontal geomagnetic field
}

\author{
P. Wintoft \\ Swedish Institute of Space Physics, Lund, Sweden \\ Received: 30 December 2004 - Revised: 31 March 2005 - Accepted: 3 May 2005 - Published: 28 July 2005
}

\begin{abstract}
The local ground geomagnetic field fluctuations $(\Delta B)$ are dominated by high frequencies and $83 \%$ of the power is located at periods of $32 \mathrm{~min}$ or less. By forming 10min root-mean-square (RMS) of $\Delta B$ a major part of this variation is captured. Using measured geomagnetic induced currents (GIC), from a power grid transformer in Southern Sweden, it is shown that the 10-min standard deviation GIC may be computed from a linear model using the RMS $\Delta X$ and $\Delta Y$ at Brorfelde (BFE: $11.67^{\circ} \mathrm{E}, 55.63^{\circ} \mathrm{N}$ ), Denmark, and Uppsala (UPS: $17.35^{\circ} \mathrm{E}, 59.90^{\circ} \mathrm{N}$ ), Sweden, with a correlation of $0.926 \pm 0.015$. From recurrent neural network models, that are driven by solar wind data, it is shown that the log RMS $\Delta X$ and $\Delta Y$ at the two locations may be predicted up to $30 \mathrm{~min}$ in advance with a correlation close to $0.8: 0.78 \pm 0.02$ for both directions at BFE; $0.81 \pm 0.02$ and $0.80 \pm 0.02$ in the $X$ - and $Y$-directions, respectively, at UPS. The most important inputs to the models are the 10-min averages of the solar wind magnetic field component $B_{z}$ and velocity $V$, and the 10-min standard deviation of the proton number density $\sigma_{n}$. The average proton number density $n$ has no influence.
\end{abstract}

Keywords. Magnetospheric physics (Solar wind - magnetosphere interactions) - Geomagnetism and paleomagnetism (Rapid time variations)

\section{Introduction}

The Earth's magnetosphere is a dynamic system that responds to changes in the upstream solar wind. Through complex processes that includes magnetic reconnection and viscous instabilities energy is transferred from the solar wind into the magnetosphere (Baumjohann and Haerendel, 1987) with subsequent energy dissipation through geomagnetic storms and substorms (Gonzalez et al., 1994). During the storm different current systems are modified, like the ionospheric currents, ring current, and magnetopause current. On the ground the currents are observed as deviations of the local

Correspondence to: P. Wintoft

(peter@lund.irf.se) geomagnetic field (Nishida, 1978). The effects of geomagnetic disturbances are observed on technological systems, such as electrical power grids, pipe lines, and telegraph lines (Boteler et al. (1998), Lundstedt ${ }^{1}$ ) and are called geomagnetically induced currents (GIC). There is great interest in modelling GIC, both for post-event analysis and for predictions. As a result there are three parallel GIC studies within the ESA Space Weather Applications Pilot Project and these can be found at the web page http://www.esa-spaceweather.net/.

The calculation of GIC can, in principle, be divided into two steps (Pirjola, 2002). The first step is the geophysical part which involves the determination of the horizontal geoelectric field at the Earth's surface. The second step is the engineering part which involves the calculation of the currents in the system based on the electric field and knowing the system layout and resistance. However, the geoelectric field is not directly available and must be estimated from the geomagnetic field. One approach is to use geomagnetic indices, as several can be successfully predicted from the solar wind, like $A E$ (Gleisner and Lundstedt, 2001a), $D_{s t}$ (Vassiliadis and Klimas, 1999; Lundstedt et al., 2002), and $K_{p}$ (Boberg et al., 2000). The index may then be translated into a physical quantity that is related to GIC. For example, Boteler (2001) showed that there is close to a linear relationship between the 3-h $K_{p}$ index and the logarithm of the ground electric field. However, the indices have their limitations because they have been derived to capture some specific aspect of the magnetospheric variation. Another approach is to compute an equivalent ionospheric current system from a measured local geomagnetic field and to assume that the geomagnetic variations at the Earth's surface can be explained by a horizontal divergence-free ionospheric current system (Viljanen et al., 2003). The method was applied using measured geomagnetic data with a temporal average of one minute and then compared to measured GIC. The relative errors were less than $30 \%$ for large GIC values. However, from a solar wind-magnetosphere coupling study it is currently not

\footnotetext{
${ }^{1}$ Lundstedt, H., The Sun, Space Weather and GIC Effects in Sweden, Adv. Space Res, in review, 2004
} 
feasible to try to model the one minute data. A straightforward solution to this is to temporally average the solar wind and geomagnetic data. For example, it is possible to predict the 10-min average local magnetic field from solar wind data (Gleisner and Lundstedt, 2001b). But, as the electric field is related to the rate-of-change of the magnetic field $(d B / d t)$ via Faraday's law of induction

$\nabla \times E=-\frac{\partial B}{\partial t}$

a more basic quantity to use is the time difference of $B$, i.e. $\Delta B(t)=B(t+1)-B(t)$. However, as will be shown in this paper, most of the power in $\Delta B$ is located at small scales (high frequencies) and therefore a large fraction of the signal will be lost if $\Delta B$ is temporally averaged, or if $\Delta B$ is formed from a temporally averaged $B$. This happens already at 5- to 10-min averages. Therefore, other moments of $\Delta B$ should be considered. In the work by Weigel et al. (2002) models were developed that predict the average absolute value of $\Delta B$ with a temporal resolution of $30 \mathrm{~min}$. More specifically, they studied the north-south component of the magnetic field, i.e. $\langle|\Delta X|\rangle_{30 \mathrm{~min}}$. The best model reached an overall prediction efficiency of 0.4 based on data from 1998-1999.

In this work the time difference of the local magnetic field is also studied but using a slightly different approach. Both the north-south $(\Delta X)$ and east-west $(\Delta Y)$ components are analysed in terms of their wavelet power spectra. Based on the analysis the 10-min root-mean-square (RMS) of $\Delta X$ and $\Delta Y$ are proposed as useful quantities for a solar windmagnetosphere study. The RMS $\Delta X$ and $\Delta Y$ are also shown to be well correlated to the 10-min RMS of measured GIC. Finally, recurrent neural networks are developed that predict the RMS $\Delta X$ and RMS $\Delta Y$ at two locations in southern Scandinavia from solar wind data, where data from the ACE spacecraft (Stone et al., 1998) have been used.

\section{Estimating the power spectrum of $\Delta X$ and $\Delta Y$}

The analysis is based on one-minute average north-south $(X)$ and east-west $(Y)$ local magnetic field components from Brorfelde (BFE) and Uppsala (UPS). As stated in the Introduction, it is more natural to study the time derivative of the magnetic field as it is related to the electric field driving GIC. The time derivative is approximated by the one-minute difference in the two directions as

$$
\begin{aligned}
& \Delta X(t)=X(t+1)-X(t) \\
& \Delta Y(t)=Y(t+1)-Y(t),
\end{aligned}
$$

where $t$ is time in minutes. However, to make the subsequent solar wind-magnetosphere coupling study feasible the level of disturbance in $\Delta X$ and $\Delta Y$ will be addressed, instead of the detailed minute-to-minute variation. To proceed, the power distribution in $\Delta X$ and $\Delta Y$ are examined with a wavelet transform. Using the wavelet transform it is possible to simultaneously examine the signal in both time and frequency similar to the windowed Fourier transform. However, the wavelet transform is capable of more accurately separating the signal in the time-frequency domain (Addison, 2002). In the following equations we will use $\Delta X$, but the same analysis is also performed on $\Delta Y$.

Using a discrete wavelet transform (DWT) the $\Delta X$ can be decomposed into signals, called details and approximation, that are associated with different scales, where the scale corresponds to a frequency band. The decomposed signals can thus be thought of as being a band-pass filtered versions of $\Delta X$.

The total power in $\Delta X$ equals the sum of the power in the details and approximation, i.e. the transformation conserves power. However, the transformation is not time invariant, i.e. the DWT of a time-shifted $\Delta X$ is not equal to the time-shifted DWT of $\Delta X$. This is an undesired property of a transform when dealing with time series data. To ensure time invariance we use a modified DWT, called the Maximum Overlap DWT (MODWT) (Percival and Walden, 2002).

We apply the MODWT (Cornish et al., 2003) using the Daubechies wavelet, of the order of, 4 on one-minute $\Delta X$ for all data in 1998 resulting in the wavelet coefficients $W_{j, t}$ (details) and $V_{t}$ (approximation), where the level is $j \in[1, J]$, $J=7$, and time is $t \in[0,525599] \mathrm{min}$. Level $j$ is associated with scale

$\tau_{j}=2^{j-1}$.

As the time resolution is one minute the scale is also in minutes. The variance, or power, at level $j$ is

$v_{j}^{2}=\frac{1}{N} \sum_{t} W_{j, t}^{2}$,

where $N=525600$ are the number of data points. As the MODWT conserves power we have

$\frac{1}{N} \sum_{t} \Delta X_{t}^{2}=\sum_{j} v_{j}^{2}+\frac{1}{N} \sum_{t} V_{t}^{2}$.

The signal at level $j$ is associated with frequencies in the range

$f_{j} \in\left[\frac{1}{2^{j+1}}, \frac{1}{2^{j}}\right]=\left[\frac{1}{4 \tau_{j}}, \frac{1}{2 \tau_{j}}\right]$.

Thus, if we compute the power spectrum $S(f)$ of $\Delta X$ with the Fourier transform, then the wavelet variance at level $j$ is approximately equal to the power in the frequency band given by $f_{j}$, according to (Percival and Walden, 2002)

$v_{j}^{2} \approx 2 \int_{1 / 2^{j+1}}^{1 / 2^{j}} S(f) d f$.

The factor 2 in front of the integral comes from the fact that $S(f)$ is mathematically defined over the interval $f \in[-1 / 2,1 / 2]$. But, as $\Delta X$ is a real value, it follows that $S(f)=S(-f)$, so that the negative frequencies can be interpreted in terms of positive and physical frequencies. In Fig. 1 the MODWT estimated power spectra for $\Delta X$ (north-south 
component) and $\Delta Y$ (east-west component) at both Brorfelde and Uppsala are shown. It is seen that the power is concentrated to small scales (high frequency). More than $83 \%$ of the power is located at the first 4 levels, corresponding to scales smaller than $\tau_{4}=2^{4-1}=8$ min or frequencies higher than $1 / 32 \mathrm{~min}^{-1}$, and $99 \%$ of the power is captured by the details up to level $j=J=7$. This means that the approximation which contains variations with scales longer than $128 \mathrm{~min}$ has a very small contribution to the total power in $\Delta X$. We also see that the total power is higher for Uppsala than for Brorfelde, as is expected for a station closer to the auroral oval. However, the spectral distributions of $\Delta X$ and $\Delta Y$ are quite different. For $\Delta X$ the power decreases monotonically with increasing scale (decreasing frequency), whereas $\Delta Y$ shows a more flat distribution for the first 4 levels. Clearly, the dynamics in the two directions are different.

A consequence of the localisation of power at high frequencies is that temporal averaging of $\Delta X$ will remove most of the variance in the signal. Thus, it is not so useful to study temporal averages of $\Delta X$. It is also worth noting that a T-minute average of $\Delta X$ will cancel all terms except the endpoints, so that it becomes equivalent to estimating the derivative using only two points, i.e. $\langle\Delta X\rangle_{T}(t)=1 / T(X(t+T)-X(t))$.

Returning to the wavelet coefficients $W_{j, t}$ we see that they represent $J$ different time series that describe variations at different scales, or frequency bands. We may form a set of new time series representing the variance over $T$ minute time windows

$v_{j}^{2}(t)=\frac{1}{T} \sum_{t^{\prime}=t}^{t+T} W_{j, t^{\prime}}^{2}$,

where we set $T=10$-min. The power conservation does not strictly hold over $10 \mathrm{~min}$ windows but the correlation is still high. Models could now be developed that predict the variance $v_{j}^{2}$ in $\mathrm{X}$ and $\mathrm{Y}$, and thereby estimate not only the magnitude of the variation in $\Delta X$ and $\Delta Y$, but also at what frequencies the disturbances are located. Currently, a study is performed that aims at modifying existing GIC models to make use of this kind of data to calculate the RMS GIC based on power grid data. ${ }^{2}$ However, in this work we will, as a first approximation, assume that the power distribution is constant over time. From Eq. (6) we can define the fractional power as

$\alpha_{j}=\frac{v_{j}^{2}}{1 / N \sum_{t} \Delta X_{t}^{2}}$,

where $\sum \alpha_{j} \approx 1$ as the last term approximation of Eq. (6) is close to zero. Next, we form 10-min mean-square (MS) $\Delta X$ as

$r^{2}(t)=\frac{1}{10} \sum_{t^{\prime}=t}^{t+9} \Delta X\left(t^{\prime}\right)^{2}$.

\footnotetext{
${ }^{2}$ Private communications: R. Pirjola, A. Pulkkinen, A. Viljanen,
}

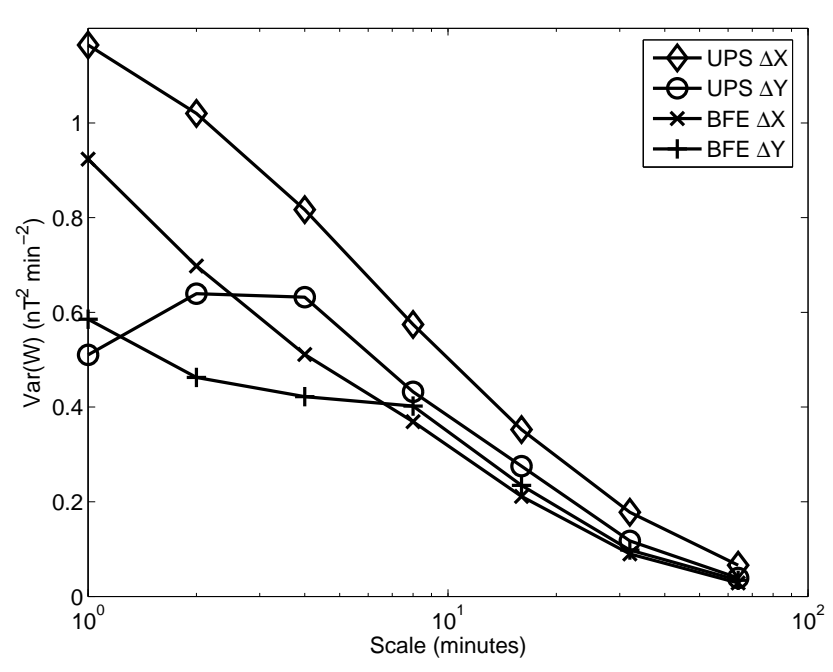

Fig. 1. The figure shows the power distribution of $\Delta X$ and $\Delta Y$ at Brorfelde (BFE), Denmark, and Uppsala (UPS), Sweden, based on 525600 one-minute data points from 1998. $\Delta X$ and $\Delta Y$ are the one-minute time differences of the north-south and east-west magnetic field components, respectively.

From Eq. (6) we see that

$r^{2}(t) \approx \sum_{j} v_{j}^{2}(t)$,

and with the assumption of a constant power distribution we obtain

$v_{j}^{2}(t) \approx \alpha_{j} r^{2}(t)$

To summarise, we may develop a model that predicts the 10 min MS, or RMS, $\Delta X$ and $\Delta Y$. Then, assuming that the power distribution is constant over time we also obtain an estimate of the power at different frequencies. Before we proceed with the solar wind $-(\Delta X, \Delta Y)$ models we study the correlation between MS $(\Delta X, \Delta Y)$ and MS GIC for a single site in Southern Sweden.

\section{Correlation between MS $\Delta X$ and $\Delta Y$, and MS GIC}

The GIC flowing between the transformer neutral and the ground has been measured at a location in Southern Sweden. The measurements have been carried out for a number of periods during the years 1998 to 2000 and the data set consists of almost 100000 one-minute samples. The measured GIC ranges from - 269 Amperes (A) to 195 A. As previously stated, with knowledge about the power grid configuration and the ground conductivity, the GIC may be computed from the time derivative of the horizontal magnetic field (Viljanen et al., 2003). Therefore, we expect to find a correlation between the MS $\Delta X$ and $\Delta Y\left(r^{2}\right)$, and the MS GIC $\left(g^{2}\right)$. Using a least-squares fit between $r^{2}$ and $g^{2}$ we find

$\hat{g}^{2}=\left(0.47+0.15 r_{1}^{2}+0.08 r_{2}^{2}+0.15 r_{3}^{2}+0.05 r_{4}^{2}\right) \mathrm{A}^{2}$, 

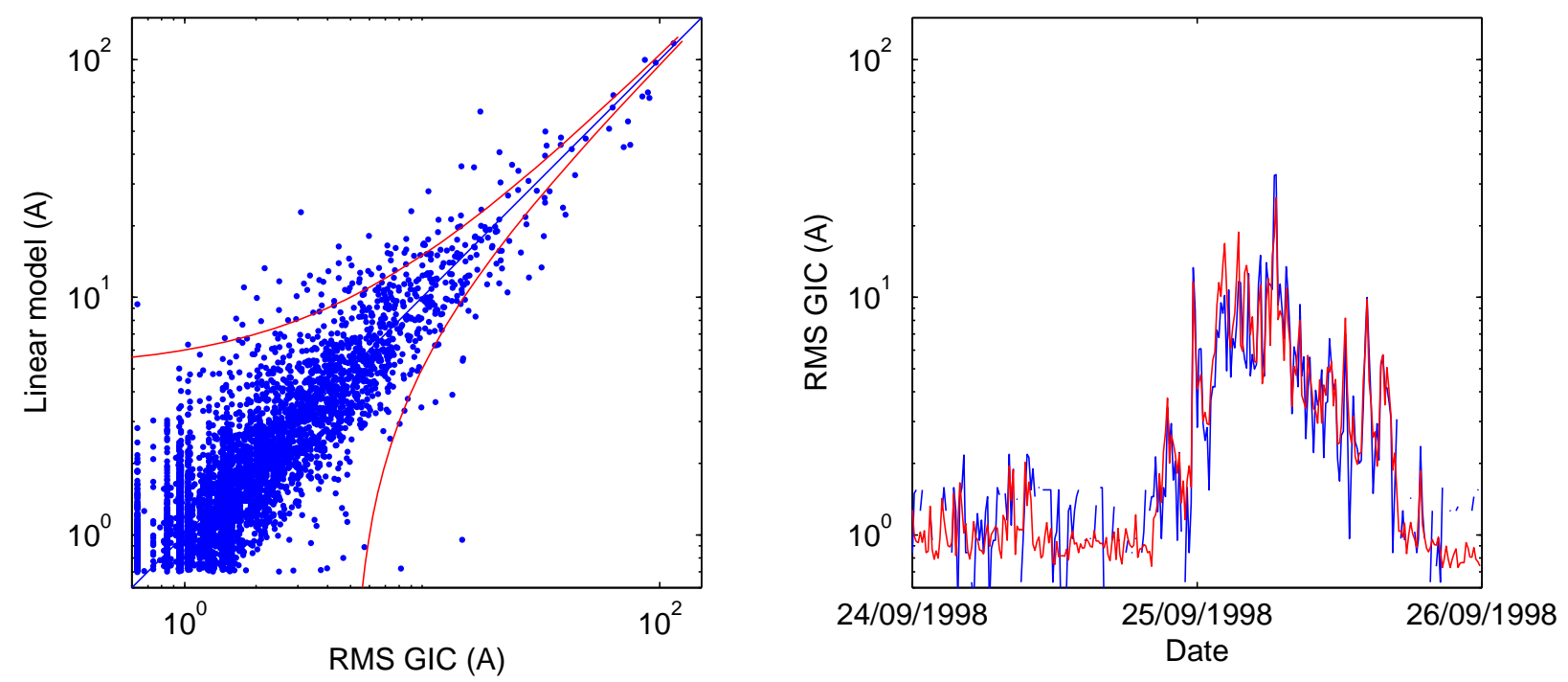

Fig. 2. The left plot in the figure shows the correlation between the 10-min RMS GIC from the linear model and the measured 10-min RMS GIC. The two curves mark the \pm 5 Amp. error. The right plot shows an example of a GIC event for the period 24 and 25 September 1998. The blue curve is the observed GIC and the red curve is the GIC from the linear model.

where $\quad r_{1}=\operatorname{RMS}\left(\triangle X_{B F E}\right), \quad r_{2}=\operatorname{RMS}\left(\triangle Y_{B F E}\right)$, $r_{3}=\operatorname{RMS}\left(\triangle X_{U P S}\right)$, and $r_{4}=\operatorname{RMS}\left(\triangle Y_{U P S}\right)$. The correlation between $g^{2}$ and $\hat{g}^{2}$ is $0.929 \pm 0.015$ at the $95 \%$ confidence level, taking into account the autocorrelation in the time series (see next section). In Fig. 2 the RMS GIC from the linear model is shown together with the measured RMS GIC. The high correlation of the single site empirical linear model indicates that it should be possible to compute the RMS GIC at other locations and for other power grid configurations using the RMS $\Delta X$ and $\Delta Y$ as inputs.

\section{Coupling of the solar wind to RMS $\Delta X$ and $\Delta Y$}

Now we turn to the solar wind - RMS $(\Delta X, \Delta Y)$ models. We will use a recurrent neural network similar to that described in Lundstedt et al. (2002). This kind of model captures the dynamics in the system that shall be modelled through internal feedback connections. One advantage of using a recurrent model over a model with time delays on the inputs is that the memory of the system need not be given explicitly. An interesting feature of the recurrent network is that it is possible to rewrite the network equation into a set of coupled differential equations that can be used for further physical interpretation.

The input to the model is the solar wind data from the ACE SWEPAM and MAG instruments (McComas et al., 1998). The 64-s plasma data and the 16-s magnetic field data are resampled to 10-min averages. In addition, the 10-min standard deviations are also used, as the average does not always give a good representation of the original data. For example, two different 10 -min intervals may have a similar average proton number density, but very different standard deviations caused by the presence of large variations around the average due to strong shocks, waves, or turbulence. The input data is collected into the vector

$\mathbf{x}(t)=\left[\mu_{B z}(t), \sigma_{B z}(t), \mu_{n}(t), \sigma_{n}(t), \mu_{V}(t), \sigma_{V}(t),\right]$

where $\mu_{\bullet}$ is the average and $\sigma_{\bullet}$ is the standard deviation of the solar wind magnetic field $B_{z}$, proton number density $n$ (hereafter called density), and velocity $V$. In order to also model any seasonal and local time variation, the sine and cosine of the fractional year and fractional local time are given at the input

$\mathbf{y}(t)=\left[\sin \frac{2 \pi \mathrm{D}}{365}, \cos \frac{2 \pi \mathrm{D}}{365}, \sin \frac{2 \pi \mathrm{L}}{24}, \cos \frac{2 \pi \mathrm{L}}{24}\right]$,

where $\mathrm{D}$ is the decimal day of the year and $\mathrm{L}$ is the local time in decimal hours. In total there are 10 inputs.

The output from the model is the predicted $\log r(t+\tau)$, where $\tau$ is the prediction horizon. Typically, the time it will take a structure in the solar wind at L1 to reach the Earth's magnetopause will vary from $30 \mathrm{~min}(800 \mathrm{~km} / \mathrm{s})$ to $80 \mathrm{~min}$ $(300 \mathrm{~km} / \mathrm{s})$. This variable prediction horizon could, in principle, be handled by either shifting the solar wind input or adjusting $\tau$ with a time lag determined by the current solar wind velocity. However, this will alter the shape of the time series and artificially modify the dynamics of the system. Therefore, we set $\tau=30 \mathrm{~min}$ and let the neural network adjust to the given situation.

As mentioned in the Introduction, any temporal averaging will decrease the forecast lead time. To illustrate this we may consider a time dependent parameter $x(t)$ that is collected with a sampling interval $\Delta t$ that results in the time series $x_{i}$. The corresponding time stamp $t_{i}$ marks the beginning of the interval so that $x_{i}$ is the average of $x(t)$ over the interval $t \in\left[t_{i}, t_{i+1}\right]$ where $t_{i+1}=t_{i}+\Delta t$. Similarly, we may have another variable $y(t)$ sampled to $y_{i}$. If we now wish to 
develop a model that predicts $y$ from $x$ with some lead time $\tau$ we have $\hat{y}(t+\tau)=f(x(t))$, where $\hat{y}$ is the prediction of $y$. This leads to the discrete model

$\hat{y}_{i+k}=f\left(x_{i}\right)$,

where $\tau=k \Delta t$. Now assume that the current time is $t_{0}$. The latest available input is $x_{-1}$ and it has been collected over the time interval $\left[t_{-1}, t_{0}\right]$. With a forecast time of $\tau=k \Delta t$ we will therefore forecast $y_{k-1}$, resulting in a true forecast time of $\tau^{\prime}=\tau-\Delta t$. In order for the model to perform actual forecasts we must have $\Delta t \leq \tau$.

The solar wind data and ground magnetic field data are extracted from the six year period 1998-2003, giving in total about 300000 10-min data samples. However, the complete data set is dominated by quiet conditions with $r$ close to zero. If the network was to be trained on this set, it would become heavily biased towards predicting quiet levels and thereby poorly predict storm levels. The optimal situation is to have a balanced data set in which all levels of disturbance occur in equal numbers. However, the optimal situation can usually not be achieved due to the distribution of data, and for recurrent networks the data must also be contiguous. Therefore, a subset is selected using the following algorithm. First, all contiguous sequences longer than $48 \mathrm{~h}$ that contain at least one event with $r>10 \mathrm{nT} / \mathrm{min}$ are selected. This results in 101 sequences with lengths ranging from $48 \mathrm{~h}$ up to $120 \mathrm{~h}$ that contain both quiet and disturbed conditions. Finally, the sequences are sorted with respect to the variance in $r$, and three independent data sets are created by selecting every third sequence. This results in about 15000 data points in each set, where each set has a similar mean and standard deviation. The three sets are used for training, validation, and testing. The training set is used for the weight adjustment, the validation set is used to determine the optimal network, and the test set is used to test the network. The input data are normalized to cover approximately the range \pm 1 and the output is log-normalized. The neural network can be summarized as

$\overline{\log r_{i}}(t+\tau)=f_{i}(\mathbf{x}(t))$,

where $\overline{\log r_{i}}$ is the output and $\tau$ is the prediction horizon. The goal of the training procedure is to change the free parameters (weights) of $f$ so that the squared error $\left(\log r_{i}(t)-\overline{\log r_{i}}(t)\right)^{2}$ is minimized. The network function $f$ contains input units, hidden units, context units, and an output unit. The units are connected with weights, and each hidden unit and output unit has a bias. The context units contain a delayed copy of the hidden units that are fed back into the hidden units; this is the recurrent layer. To a first approximation, the recurrent layer is an exponential trace memory, where the weights represent the decay terms. Thus, the context units contain the memory of the system.

The weights are initialised to small random values and then the network is trained. Typically, both the training set error and the validation set error decrease during the first part of the training phase as the network adjusts to general features in the data. Then, as training continues the training error still decreases, while the validation error may occasionally increase, passing through several local minima. Finally, the validation error just continues to grow while the training error still decreases. The values of the weights at which the network reached the deepest validation minimum are considered to be the optimal weight. During the first phase the network adjusts to general features in the data, then it picks out more detailed features but also starts to adjust to the noise in the data, and then finally the network continues to adjust to the remaining noise. By monitoring the progress of the validation set error, we can thus find the optimal network.

A large number of networks with different architectures are trained to predict $\log r_{i}$, and the optimal network is determined using the validation set. The initial network is fully connected and has 10 inputs, $n_{h}$ hidden units, $n_{c}=n_{h}$ context units, and one output. As the output unit and each hidden unit also has a bias, the total number of weights is $n=10 n_{h}+n_{h}+n_{c} n_{h}+n_{h}+1=1+12 n_{h}+n_{h}^{2}$. The number of hidden units is varied over $n_{h}=2,3,4,5,6$, giving networks with $n=29,46,65,86,109$ weights.

Starting with the model for the RMS $\Delta X$ at Uppsala we see that the maximum correlation is obtained for a network with $n_{h}=5$ hidden units (Fig. 3, upper left plot). The confidence limits are shown at the $95 \%$ level. In computing the correlation and confidence limits we use all three data sets: training set, validation set, and test set. There are almost 40000 data points but the autocorrelation in both the observed series and the predicted series do not fall off to zero quickly. Therefore, the effective number of independent observations (Quenouille, 1952; von Storch and Zwiers, 1999) is reduced by a factor of about 35 , giving slightly more than 1000 independent points. In Fig. 3 the horizontal line indicates the level at which the correlation is significantly lower than the highest correlation. This means that all models with a correlation above the line perform equally well, but any model falling below the line performs significantly poorer. Thus, it can be seen that there is a significant increase in the correlation going from 2 hidden units to 3 hidden units, and increasing the number of hidden units has very little (or no) effect. Similar results are obtained for UPS $\Delta Y$, and BFE $\Delta X$ and $\Delta Y$.

It is interesting to see which inputs have an influence on the model. It is not possible to merely look at the strengths of the weights to judge which inputs are of importance. Instead, each weight must be removed and the change in performance monitored. For large networks there are more efficient ways to achieve this, in which the second derivative of the error with respect to the weights is computed (Le Cun et al., 1990). But since the network used here is quite small, we may simply remove one input at a time and compute the change in error. Before the error is computed the network is additionally trained for a few iterations, so that the remaining weights may be altered to compensate for a possible change in bias. The initial network is fully connected with 10 inputs and 5 hidden units. When one input is removed the total number of weights is decreased by 5 . After removing one input at a 

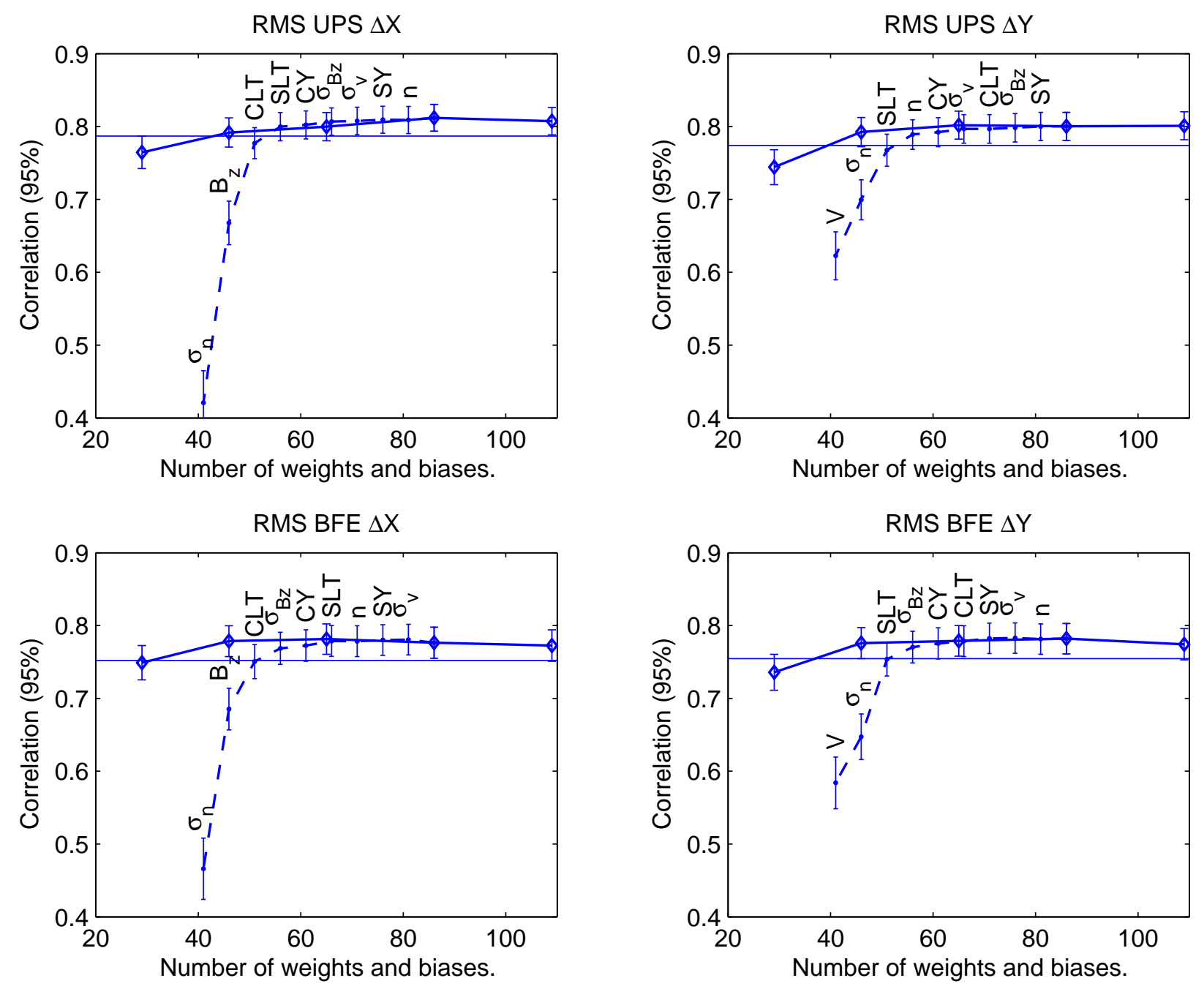

Fig. 3. The figure shows the correlation coefficients $C(\log r, \overline{\log r})$ for networks with different numbers of weights and biases. The plots show the results for the models, predicting the 10-min root-mean-square (RMS) $\Delta X$ (RMS UPS $\Delta X$ ) and RMS $\Delta Y$ (RMS UPS $\Delta Y$ ) at Uppsala, and RMS $\Delta X$ (RMS BFE $\Delta X$ ) and $\Delta Y$ (RMS BFE $\Delta Y$ ) at Brorfelde. The error bars indicate the $95 \%$ confidence levels. The horizontal line in each plot indicates the level at which the correlation is significantly lower than the highest correlation. The solid curve connected with diamonds corresponds to the fully connected networks with 2,3, 4, 5 and 6 hidden units. The labels along the dashed curve show which input that has been removed. The labels have the following meaning: solar wind magnetic field $\mathrm{z}$-component $\left(B_{z}\right)$, proton number density (n), and velocity (V); standard deviations of $B_{z}\left(\sigma_{B z}\right), \mathrm{n}\left(\sigma_{n}\right)$, and V $\left(\sigma_{V}\right)$; sine (SY) and cosine (CY) of the year; sine (SLT) and cosine (CLT) of local time.

time we will have a set of 10 different models, each having 9 inputs. The model with the highest correlation is chosen from the set to be used for continued pruning. The process is repeated until there is only one input unit left. The network pruning results in the change in correlation according to the points connected with dashed lines in Fig. 3. Each label indicates which input has been removed. The procedure is repeated for Uppsala $\Delta Y$, and Brorfelde $\Delta X$ and $\Delta Y$. For all models the following inputs have no influence: sine and cosine of the year, standard deviations of $B_{z}$ and velocity $V$, and density $n$. Then there are some differences between the models. In both Uppsala and Brorfelde the $\Delta X$-models show a weak dependence on the cosine local time (CLT). Looking at the local time distribution of $\Delta X$ it follows a cosine func- tion with a maximum around noon and a minimum around midnight. Further pruning reveals that the most important inputs, ordered in increasing importance, are $B_{z}, \sigma_{n}$, and $V$. Now studying the $\Delta Y$-models we note that there is a weak coupling to sine local time instead of cosine. Again, looking at the local time distribution there is a maximum in the morning sector that can be described by a sine curve, however, the distribution in the afternoon sector does not follow the sine shape, instead it levels out, showing no variation in local time. Ordering the remaining inputs in increasing importance we find $\sigma_{n}, V$, and $B_{z}$. Apart from the difference in local time distribution, there also seems to be a difference in the coupling to the solar wind between $\Delta X$ and $\Delta Y$. The two most important parameters for $\Delta X$ are $V$ and $\sigma_{n}$, and they are 

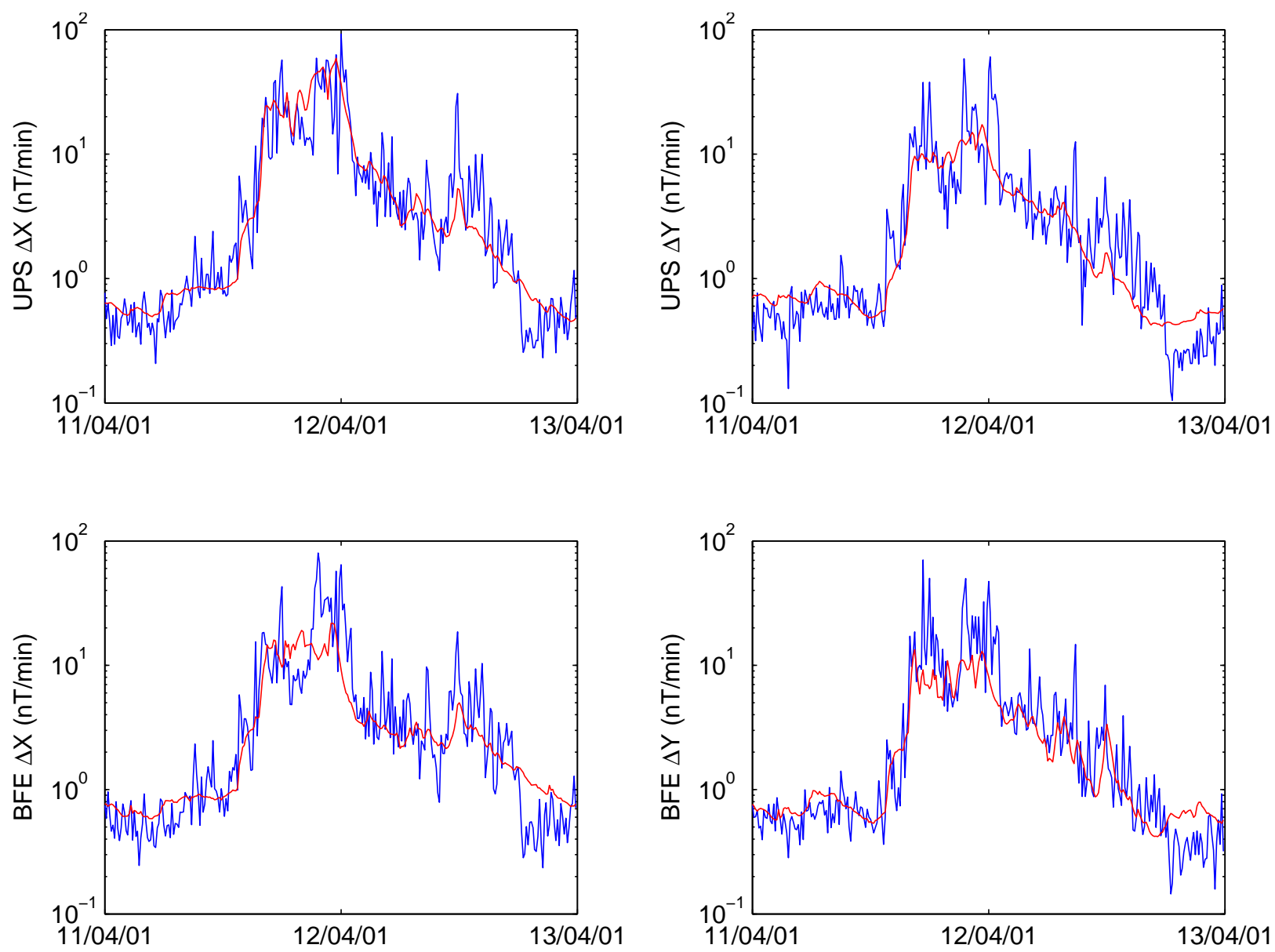

Fig. 4. The four plots show the observed (blue) and predicted (red) 10-min RMS $\Delta X$ and $\Delta Y$ at Uppsala (UPS) and Brorfelde (BFE) during a storm in 11-12 April 2001. The correlation in this case is around 0.90. The event was selected from the test set.

related to pressure variations in the solar wind that compress the dayside magnetopause. This is also consistent with the local time variation seen in $\Delta X$. On the other hand, for $\Delta Y$ the two most important parameters are $B_{z}$ and $V$ that may be interpreted to be more linked to the reconnection process at the magnetopause which causes sub-storms and storms.

As previously mentioned, the prediction lead time is $30 \mathrm{~min}$. We may examine if it is possible to increase the lead time without degrading the performance of the model. We increase the lead time in steps, with continued training of the network, and compute the correlation. It turns out that the correlation for both $\Delta X$ and $\Delta Y$ monotonically decreases, even though we may extend the lead time to 70$90 \mathrm{~min}$ before it becomes significantly poorer. However, the $\Delta X$-model shows a steeper decrease in correlation than the $\Delta Y$-model. This is consistent with the finding above, that solar wind pressure variations are more important for $\Delta X$ than $\Delta Y$, and that the substorm process dominates the $\Delta Y$ variations. The magnetopause current responds directly to solar wind pressure changes, so the only available lead time is the travel time from L1 to the Earth's magnetopause. On the other hand, there are additional time delays before the substorm develops after the southward turning of $B_{z}$.

\section{Discussion}

As also shown by Weigel et al. (2002), we found no coupling from solar wind density $n$ to RMS $\Delta X$. In solar wind coupling studies the density usually enters into the equations through the dynamic pressure $p=m n V^{2}$, either as the square root of $p$ or as a linear function of $p$ (Baker, 1986). If we assume that the geomagnetic field $X$ is proportional to $p$, we have

$X \propto p \propto n V^{2}$.

Differentiating with respect to time $t$ we obtain

$\frac{d X}{d t} \propto \frac{d n}{d t} V^{2}+2 n V \frac{d V}{d t}$.

Analysing ACE data from 1998 we find that the first term in the above equation completely dominates, leaving us with an equation that does not contain $n$. In addition, $d n / d t$ is related to $\sigma_{n}$ and from the neural network we also find a dependence on $\sigma_{n}$.

The overall correlation of the models is 0.79 and the prediction efficiency (PE) (Detman and Vassiliadis, 1997) is 
0.63 for the logarithm of the 10-min RMS data. Transforming the data to 10-min RMS values the correlation drops to 0.71 and the PE to 0.50. It is difficult to make a comparison with the Weigel et al. (2002) models, as they predict the 30min average of the absolute value $|\Delta X|$ at higher latitudes. However, forming 30-min RMS the correlation reaches 0.77 and the PE 0.58 .

Another issue is that the variance in RMS $\Delta X$ is much larger than the variance in $|\Delta X|$. The variance of the one-minute $\Delta X$ at Uppsala for 1998 is $\sigma^{2}(\Delta X)=4.23$ and the variance of the 10-min RMS $\Delta X$ is $82 \%$ of that, or $\sigma^{2}(\operatorname{RMS} \Delta X)=3.45$. The 10-min average $|\Delta X|(\mu(|\Delta X|))$ has a variance of only $\sigma^{2}(\mu(|\Delta X|))=2.30$ which corresponds to $55 \%$ of the original signal.

We may now look at an example of a prediction. The event is chosen from the test set and extends over two days in April 2001. The four plots in Fig. 4 show the observed RMS $\Delta X$ and $\Delta Y$ in blue and the predicted in red for Uppsala $\Delta X$ (upper left), Uppsala $\Delta Y$ (upper right), Brorfelde $\Delta X$ (lower left), and Brorfelde $\Delta Y$ (lower right). The units are in $\mathrm{nT} / \mathrm{min}$. For both locations, and both directions, the RMS values increase from about $0.5 \mathrm{nT} / \mathrm{min}$ to $20 \mathrm{nT} / \mathrm{min}$ and with individual peaks close to $100 \mathrm{nT} / \mathrm{min}$. The evolution of the four time series are quite similar, but there are also some smaller differences. We see from the plots that the models predicts the general features well but not the sample to sample variations.

The models have been implemented for real time operation and can be found at the web page http://www.lund.irf.se/ gicpilot/gicforecastprototype. The forecasts are updated every $10 \mathrm{~min}$ and produces a plot of the RMS $\Delta X$ and $\Delta Y$ for Uppsala and Brorfelde. The linear model given by Eq. (14) has also been implemented to produce RMS GIC forecasts.

\section{Conclusions}

The main purpose of this work was to develop a model that is capable of forecasting $d X / d t$ and $d Y / d t$ in Southern Sweden. Two magnetic observatories lying at the southwest (Brorfelde) and northeast (Uppsala) corners of the area under consideration were selected. The distance between the two sites is about $600 \mathrm{~km}$. At this stage we do not try to predict the one minute $\Delta X$ or $\Delta Y$ as the time series are dominated by fluctuations that are not directly coupled to the solar wind. Instead, we found that using the mean-square (MS) or root-mean-square (RMS) of $\Delta X$ and $\Delta Y$ formed over $10 \mathrm{~min}$ captures a large fraction of the variance in the signal.

Analysing the importance of the input parameters it was found that there was a weak dependence on local time and that the solar wind magnetic field $B_{z}$, velocity $V$, and standard deviation $\sigma_{n}$ of the density were the most important. It was also found that there might be a slight difference in the solar wind $-\Delta X$ coupling compared to the $\Delta Y$ coupling. The former shows a stronger coupling to $\sigma_{n}$ and $V$, while the latter has a stronger coupling to $B_{z}$ and $V$.
From measured GIC at a single location we found that the 10-min RMS GIC can be quite accurately estimated from RMS $\Delta X$ and $\Delta Y$ from two nearby magnetic observatories. Therefore, we believe that it should be possible to use forecasted RMS $\Delta X$ and $\Delta Y$ as a general indicator of the GIC level.

In future work the models should be developed to directly predict the variance $v_{j}^{2}(t)$ at different levels $j$. It also has to be seen whether this will significantly improve the estimates of the variation in $\Delta X$ and $\Delta Y$ and thereby also give further insight into the solar wind-magnetosphere coupling.

Acknowledgements. The author is grateful to the IMAGE network and the Danish Meteorological Institute for providing the magnetometer data. The ACE team is acknowledged for providing the solar wind plasma (SWEPAM) and magnetic field (MAG) data. Elforsk $\mathrm{AB}$ is acknowledged for providing the GIC data.

This work has been partly funded by the ESA/ESTEC Contract No. 16953/02/NL/LvH and the Swedish National Space Board.

Topical Editor R. Forsyth thanks H. Fichtner and another referee for their help in evaluating this paper.

\section{References}

Addison, P. S.: The Illustrated Wavelet Transform Handbook, Institute of Physics Publishing, 2002.

Baker, D. N.: Statistical analyses in the study of solar windmagnetosphere coupling, Solar Wind-Magnetosphere Coupling, 17-38, 1986.

Baumjohann, W. and Haerendel, G.: Entry and dissipation of energy in the Earth's magnetosphere, in Space Astronomy and Solar System Exploration: Proceeding of summer school held at aplbach, Austria, 29 July-8 August, 1986, 121-130, ESA, 1987.

Boberg, F., Wintoft, P., and Lundstedt, H.: Real time $K_{p}$ predictions from solar wind data using neural networks, Physics and Chemistry of the Earth, 25, 275-280, 2000.

Boteler, D.: Assessment of geomagnetic hazards to power systems in Canada, Natural Hazards, 23, 101-120, 2001.

Boteler, D. H., Pirjola, R. J., and Nevanlinna, H.: The effects of geomagnetic disturbances on electrical systems at the Earth's surface, Adv. Space Res., 22, 17-27, 1998.

Cornish, C. R., Percival, D. B., and Bretherton, C. S.: The WMTSA Wavelet Toolkit for Data Analysis in the Geosciences, 84, fall Meet. Suppl., Abstract NG11A-0173, 2003.

Detman, T. R. and Vassiliadis, D.: Review of techniques for magnetic storm forecasting, 253-266, AGU, 1997.

Gleisner, H. and Lundstedt, H.: Auroral electrojet predictions with dynamic neural networks, J. Geophys. Res., 106, 24 541-24 550, $2001 \mathrm{a}$.

Gleisner, H. and Lundstedt, H.: Neural network-based local model for prediction of geomagnetic disturbances, J. Geophys. Res., 106, 8425-8433, 2001b.

Gonzalez, W. D., Joselyn, J. A., Kamide, Y., Kroehl, H. W., Rostoker, G., Tsurutani, B. T., and Vasyliunas, V. M.: What is a geomagnetic storm?, J. Geophys. Res., 99, 5771-5792, 1994.

Le Cun, Y., Denker, J., and Solla, S.: Optimal brain damage, in Neural information processing systems, (Ed.) Touretzky, D. S., 2, 598-605, Morgan Kaufmann, San Mateo, Denver, 1990. 
Lundstedt, H., Gleisner, H., and Wintoft, P.: Operational forecasts of the geomagnetic Dst index, Geophys. Res. Let., 29, 34-1-344, 2002.

McComas, D. J., Bame, S. J., Barker, P., Feldman, W. C., Phillips, J. L., Riley, P., and Griffee, J. W.: Solar wind electron proton alpha monitor (SWEPAM) for the Advanced Composition Explorer, Space Sci. Rev., 86, 563-612, 1998.

Nishida, A.: Geomagnetic Diagnosis of the Magnetosphere, 9 of Phys. Chem. Space, Springer-Verlag, 1978.

Percival, D. B. and Walden, A. T.: Wavelet methods for time series analysis, Cambridge University Press, 2002.

Pirjola, R.: Review on the calculation of surface electric and magnetic fields and of geomagnetically induced currents in groundbased technological systems, Surv. Geoph., 23, 71-90, 2002.

Quenouille, M. H.: Assoicated measurements, Butterworths Scientific Publications, 1952.
Stone, E. C., Frandsen, A. M., Mewaldt, R. A., Christian, E. R., Margolies, D., Ormes, J. F., and Snow, F.: The Advanced Composition Explorer, Space Sci. Rev., 86, 1, 1998.

Vassiliadis, D. and Klimas, A. J.: The $D_{s} t$ geomagnetic response as a function of storm phase and amplitude and the solar wind electric field, J. Geophys. Res., 104, 24 957-24 976, 1999.

Viljanen, A., Pulkkinen, A., Amm, O., Pirjola, R., Korja, T., and BEAR Working Group: Fast computation of the geoelctric field using the method of elementary current systems and planar Earth models, Ann. Geophys., 22, 101-113, 2004,

SRef-ID: 1432-0576/ag/2004-22-101.

von Storch, H. and Zwiers, F. W.: Statistical analysis in climate research, Cambridge University Press, 1999.

Weigel, R. S., Vassiliadis, D., and Klimas, A. J.: Coupling of the solar wind to temporal fluctuations in ground magnetic fields, Geophys. Res. Let., 29, 21-1-4, doi:1029/2002GL014740, 2002. 